\title{
Manifestações imaginológicas distintas na displasia cemento-óssea florida
}

\author{
Distinct imaging manifestations in florid cemento-osseous dysplasia
}

\author{
Tamara Fernandes de Castro* \\ Lilian Cristina Vessoni Iwaki* \\ Neli Pieralisi*** \\ Mariliani Chicarelli da Silva**** \\ Elen de Souza Tolentino ${ }^{* * * *+}$
}

\section{Resumo}

Objetivo: o objetivo do presente trabalho é relatar dois casos clínicos de pacientes com displasia cemento-óssea florida (DCOF) com características distintas no exame de tomografia computadorizada de feixe cônico (TCFC). Relato de caso: no primeiro caso, paciente do gênero feminino, melanoderma, 49 anos de idade com lesões hipodensas assintomáticas distribuídas por toda a mandíbula, sugestivas de DCOF em estágio imaturo. No segundo caso, uma paciente do gênero feminino, melanoderma de 48 anos de idade, as lesões apresentavam-se hiperdensas com halo hipodenso, em mandíbula localizada bilateralmente, assintomáticas, caracterizando o estágio maduro da DCOF. Em ambos os casos, o diagnóstico de DCOF foi estabelecido por meio das imagens de TCFC associadas às características clínicas das pacientes. Nenhum tratamento foi instituído, apenas o controle periódico. Considerações finais: os casos clínicos apresentados ressaltam a importância da TCFC no diagnóstico das lesões fibro-ósseas, que, como ilustrado, podem apresentar características imaginológicas bastante distintas. Por possuir um amplo espectro de apresentações e ser encontrada em exames de imagens realizados para outros fins, a DCOF pode levar o cirurgião dentista à tomada de decisões precipitadas e, muitas vezes, condutas inadequadas, visto que procedimentos cirúrgicos são contraindicados nesses casos.

Palavras-chave: Doenças maxilomandibulares. Tomografia computadorizada de feixe cônico. Cavidade bucal.

\section{Introdução}

A displasia cemento-óssea florida (DCOF) consiste em uma lesão fibro-óssea benigna não neoplásica dos maxilares ${ }^{1-2} \mathrm{em}$ que o osso é substituído por um tecido fibroso semelhante ao cemento ${ }^{3}$, a qual pode ocorrer em áreas dentadas ou nã $0^{4-6}$. Dentes associados à DCOF não são deslocados, não apresentam reabsorção radicular ou alterações em vitalidade pulpar ${ }^{6}$. A etiologia dessa patologia é desconhecida e apresenta-se totalmente assintomáti$\mathrm{ca}^{1-2,6-7} \mathrm{e}$, em muitos casos, as lesões são detectadas em uma radiografia tomada para outros fins ${ }^{3-5,7-10}$. Tal doença possui maior expressão no gênero feminino, em pacientes melanodermas de meia idade ${ }^{2-8,10}$. Microscopicamente, essas lesões são compostas de trabéculas ósseas anastomosadas e camadas de calcificações cementoides embutidas em um tecido fibroblástico ${ }^{7,9}$. Nos exames radiográficos, a DCOF tem um amplo espectro de apresentações, no qual as lesões variam de totalmente radiolúcidas (fase osteolítica, na qual o tecido ósseo normal é substituído por um tecido fibroso com fibras colágenas e distribuição aleatória de células) ${ }^{6}$, mistas a radiopacas, quando há formação de aglomerados irregulares de tecido mineralizado e um aumento de tamanho nas trabéculas ósseas ${ }^{6,10}$, tendendo para a simetria, com localização bilateral, sendo mais comuns na mandíbula do que na maxila ${ }^{5}$. As lesões podem promover expansão das corticais ósseas e, normalmente, apresentam uma relação familiar ${ }^{3,6-7}$.

Residente do Programa de Pós-Graduação, nível especialização, na área de Radiologia Odontológica e Imaginologia, no Departamento de Odontologia, da Universidade Estadual de Maringá, Maringá, Paraná, Brasil.

Professora Associada da área de Radiologia Odontológica do Departamento de Odontologia, da Universidade Estadual de Maringá, Maringá, Paraná, Brasil.

Professora Associada da área de Radiologia Odontológica do Departamento de Odontologia, da Universidade Estadual de Maringá, Maringá, Paraná, Brasil.

Professora Associada da área de Radiologia Odontológica do Departamento de Odontologia, da Universidade Estadual de Maringá, Maringá, Paraná, Brasil.

* Professora Colaborativa da área de Radiologia Odontológica do Departamento de Odontologia, da Universidade Estadual de Maringá, Maringá, Paraná, Brasil. 
A DCOF pode se assemelhar a lesões como a osteomielite crônica esclerosante difusa (OCED), doença óssea de Paget, fibroma cemento-ossificante e displasia fibrosa ${ }^{2,10}$, doenças que requerem intervenção terapêutica e/ou cirúrgica. Em pacientes com DCOF assintomáticos o tratamento consiste apenas no acompanhamento clínico e radiográfico ${ }^{3,10}$. Nesse contexto, exames tridimensionais de imagem como a tomografia computadorizada de feixe cônico (TCFC) tornam-se ferramentas valiosas no diagnóstico da DCOF, uma vez que permitem a visualização das áreas afetadas em reconstruções axiais, coronais e sagitais ${ }^{9,10}$, sem sobreposição de estruturas e com ótima resolução. Considerando que procedimentos cirúrgicos como as biópsias devem ser evitadas em áreas afetadas ${ }^{3,5-6}$ devido à susceptibilidade do osso a infecções ${ }^{2-3}$ e que o estabelecimento do diagnóstico definitivo da DCOF na maioria dos casos é realizado em exames de imagem, o uso da TCFC pode ser fundamental.

Considerando que a DCOF é uma alteração comum dos maxilares e a dificuldade do cirurgião dentista em identificá-la e conduzi-la, o objetivo do presente trabalho é relatar dois casos clínicos de pacientes com DCOF em diferentes fases, com características distintas no exame de TCFC.

\section{Relato de caso}

Ambos os pacientes assinaram o Termo de Consentimento Livre e Esclarecido, autorizando a divulgação das informações e imagens contidas nos casos clínicos.

\section{Caso 1}

Paciente do gênero feminino, melanoderma, 49 anos de idade, foi encaminhada para exame de TCFC devido à presença de múltiplas áreas radiolúcidas distribuídas por toda mandíbula identificada em radiografia panorâmica realizada para início de tratamento odontológico (Figura 1). A paciente era sistematicamente saudável e os exames intra e extrabucal não apontaram anormalidades, com ausência de sinais clínicos de inflamação nas mucosas e de tumefações palpáveis.

A TCFC, com cortes axiais de $0,2 \mathrm{~mm}$ de espessura com intervalos de $1 \mathrm{~mm}$ e FOV de 7,5 x 14,5 $\mathrm{cm}$, evidenciou múltiplas áreas hipodensas, ora uni ora multiloculares, em região de sínfise, parassínfise e corpo de mandíbula com localização bilateral. Em alguns pontos, haviam focos de mineralização e adelgaçamento e discreta expansão das corticais vestibular e lingual. As lesões mediam entre 18 e $28 \mathrm{~mm}$, aproximadamente. A lesão em região posterior direita se estendia à base da mandíbula. Os canais mandibulares, embora envolvidos pelas áreas osteolíticas, encontram-se preservados (Figura 2). Todos os dentes inferiores apresentavam vitalidade pulpar.
Frente às características clínicas da paciente, à ausência de sintomatologia e aos achados imaginológicos, o diagnóstico foi de DCOF, sendo o cirurgião-dentista responsável e a paciente orientados a realizar controles clínico-radiográficos periódicos (a cada 6 meses), assim como a realizar manutenção de higiene oral a fim de evitar possíveis infecções.
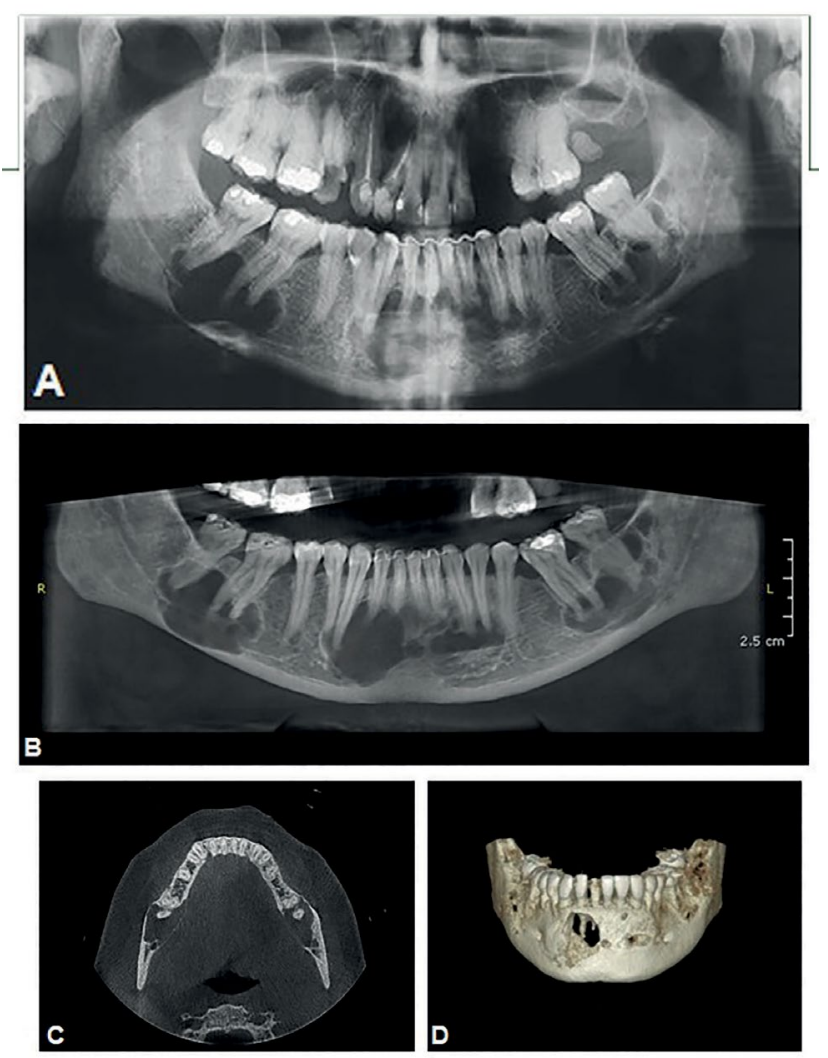

Figura 1 - Exames Imaginológicos. A. Radiografia panorâmica inicial. B. Reconstrução coronal panorâmica expondo nitidamente os limites das lesões. C. Reconstrução Axial. D. Imagem 3D

Fonte: dos autores.

\section{Caso 2}

Paciente do gênero feminino, melanoderma, 48 anos de idade, foi encaminhada_para um serviço de Estomatologia para investigação e tratamento de lesões intraósseas observadas pelo cirurgião dentista em radiografias prévias. Foi realizada a TCFC com cortes axiais de $0,2 \mathrm{~mm}$ de espessura e intervalos de $1 \mathrm{~mm}$ entre cortes, com FOV de 7,5 x 14,5 $\mathrm{cm}$. As reconstruções evidenciaram múltiplas áreas hiperdensas irregulares envoltas por halo hipodenso em corpo de mandíbula com localização bilateral. Os dentes 36 e 46 ausentes. Em alguns pontos, presença de discreto adelgaçamento de corticais lingual e vestibular. As lesões mediam entre 5 e 16 $\mathrm{mm}$, aproximadamente e os canais mandibulares encontram-se preservados. Os dentes 37 e 47 apresentavam tratamento endodôntico.

Considerando a ausência de sinais clínicos e sintomas, o diagnóstico baseado na TCFC foi de DCOF. A paciente foi orientada a realizar controles clínico- 
-radiográficos anuais, uma vez que as lesões apresentavam um padrão de imagem bastante clássico e já em fase madura. Adicionalmente, orientação quanto à saúde periodontal e tratamentos restauradores foram realizados.
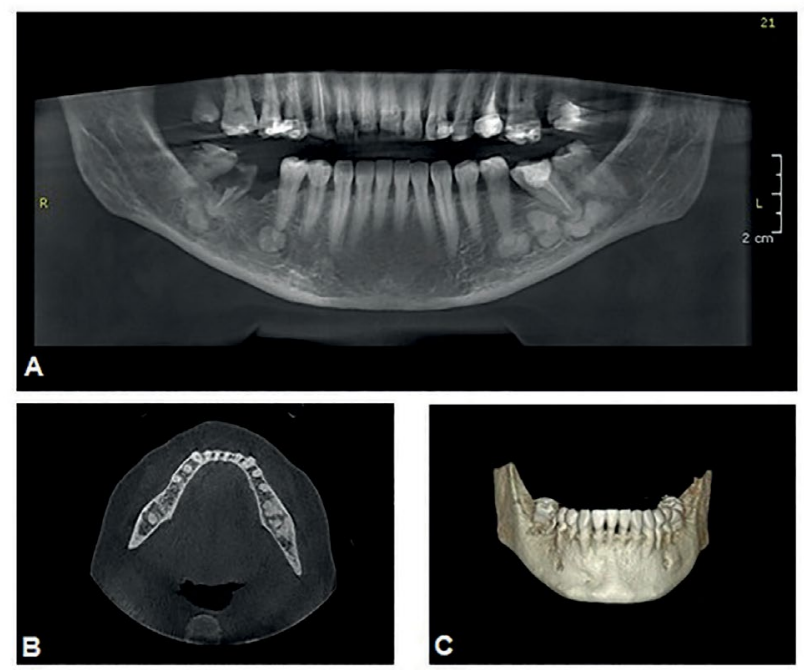

Figura 2 - TCFC do segundo caso

Legenda: $A$ - reconstrução coronal panorâmico mostrando a precisão das lesões; B - reconstrução axial; C - imagem 3D.

Fonte: dos autores.

\section{Discussão}

A DCOF é uma condição mais relatada em mulheres afrodescendentes de meia idade ${ }^{1,4,6-9}$, com idade média entre 47 e 49 anos $^{2}$, na maioria das vezes indolor e detectada em radiografias realizadas para outros propósitos, características que foram encontradas nos casos apresentados. A DCOF pode apresentar-se com um padrão de herança autossômica dominante, mas apenas em alguns relatos na literatura o padrão familiar foi confirmado ${ }^{1,5,7,9}$. Nos presentes casos, nenhum aspecto familiar pode ser estabelecido.

A etiopatogênese de DCOF não é bem estabelecida $^{6,8,10}$, sendo importante considerar suas características clínicas e radiográficas para diferenciá-la de outras lesões, como a doença óssea de Paget, OCED, fibroma cemento-ossificante e displasia fibrosa ${ }^{2,10}$. Radiograficamente, a doença de Paget pode manifestar-se como áreas radiopacas múltiplas com aparência de "algodão". Essa condição afeta o osso de todo a mandíbula e há perda de lâmina dura nos dentes envolvidos, enquanto que, na DCOF, as alterações estão centradas acima do canal mandibular ${ }^{9}$ e não há envolvimento dentário ${ }^{2,7,10}$. A doença de $\mathrm{Pa}$ get é, muitas vezes, poliostótica, envolvendo outros ossos como vértebras, fêmur, crânio, pelve e esterno, produzindo alterações bioquímicas, elevados níveis de fosfato sérico ${ }^{4,7-10}$. A DCOF é exclusiva dos ossos gnáticos e não produz alterações bioquímicas ${ }^{5}$.

A osteomielite esclerosante difusa é uma inflação primária mais comum na mandíbula que apre- senta episódios cíclicos de dor e inchaço e mostra-se como uma área de esclerose difusa, contendo pequenos focos osteolíticos mal definidos nas radiografias $^{8-9}$. Quando se torna crônica (OCED), envolve o corpo da mandíbula, do alvéolo à borda inferior e pode se estender até o ramo mandibular. Já a DCOF é vista como lesões multiloculares, redondas ou lobuladas em áreas dentadas ou nã $0^{9-10}$. Além disso, a DCOF é, frequentemente, associada a mulheres negras, enquanto a OCED é vista predominantemente em homens caucasianos adultos.

O fibroma cemento-ossificante é uma lesão neoplásica que exibe expansão de corticais vestibular e lingual ${ }^{8}$, mais grave que a $\mathrm{DCOF}^{10}$, uma vez que requer intervenção cirúrgica. Em relação à displasia fibrosa, radiograficamente ela apresenta característica patognomônica que é a aparência óssea de vidro despolido ${ }^{4,10}$, além de frequentemente expandir corticais ósseas. Todavia, em alguns casos, apenas a radiografia panorâmica pode não ser suficiente para estabelecer o diagnóstico. Nesse aspecto, a TCFC pode ser usada na diferenciação de outras lesões com aparência esclerótica semelhante à DCOF em radiografias convencionais ${ }^{9-10}$, uma vez que proporciona imagens nos três planos espaciais, identificando a localização precisa, envolvimentos de estruturas anatômicas e a extensão da lesão $0^{9}$.

O diagnóstico da DCOF é baseado principalmente nos achados clínicos-anamnésicos associados aos exames imaginológicos, não havendo necessidade, na maioria dos casos, de biópsia para o diagnóstico dessa doença ${ }^{3-4,6,8}$. Nos casos apresentados, a biópsia não foi realizada uma vez que as pacientes eram assintomáticas. Embora alguns autores tenham relatado tratamento ortodôntico ${ }^{4}$, instalação de implantes ${ }^{1}$ ou outros procedimentos cirúrgicos como ressecção para fins estéticos ${ }^{7}$, em osso displásico sem ocorrência de complicações, procedimentos cirúrgicos devem ser evitados sempre que possível, pois exodontias, biópsias ósseas e implantes dentários ${ }^{3,5-6} \mathrm{em}$ áreas com DCOF têm sido associados à ocorrência de osteomielites, sendo essas as principais complicações da $\mathrm{DCOF}^{3}$, podendo levar à fratura do maxilar ${ }^{6,8-10}$. A susceptibilidade do osso a infecções ocorre uma vez que as áreas de mineralização se apresentam extremamente densas, avasculares e com pouca capacidade de remodelação, predispondo a úlceras na mucosa, exposição e sequestros ósseos ${ }^{2,3,10}$.

Apesar de tratar-se de uma patologia comum nos maxilares, muitos cirurgiões dentistas têm dificuldade em identificar e conduzir casos de lesões fibro-ósseas, apresentando um diagnóstico incorreto ou mesmo manifestando negligência com as lesões. Nessas situações, condutas inadequadas e iatrogênicas podem ocorrer, como terapias endodônticas desnecessárias ${ }^{7,9}$, extrações dentárias, biópsias intraósseas ${ }^{3,5-6} \mathrm{e}$ outros procedimentos cirúrgicos em osso alterado, levando a prejuízos aos pacientes.

Por esse motivo, nenhum tratamento é estabelecido em casos de DCOF assintomáticos, apenas 
acompanhamento clínico-radiográfico ${ }^{3}$, como nos casos relatados, considerando-se que há um bom prognóstico. Para os pacientes sintomáticos acometidos por osteomielite crônica secundária, a antibioticoterapia aliada à curetagem pode ser necessária ${ }^{3}$.

A importância dos casos relatados dá-se pela diferença em suas manifestações nos exames de imagem. Quando as lesões estão na fase mista ou completamente radiopaca/hiperdensa, como no caso 2 , o diagnóstico é facilitado e denota um quadro mais maduro e estabilizado, além de mais característico. Entretanto, o caso 1 ilustra lesões imaturas e ativas, ainda em fase osteolítica com substituição de osso por tecido fibroso. Nesse estágio, o diagnóstico é dificultado, uma vez que o aspecto radiolúcido/ hipodenso aumenta o espectro de diagnósticos diferenciais, que inclui cistos e tumores odontogênicos. A ausência de sinais clínicos e sintomas, dentes hígidos ou com restaurações superficiais com vitalidade pulpar e as características da paciente, como idade e etnia, reforçaram a hipótese diagnóstica de DCOF. Nesse caso, o acompanhamento clínico-radiográfico em períodos mais curtos é indicado, até que as lesões calcifiquem e estabilizem.

Considerando as possíveis complicações da DCOF, a prevenção é de grande importância, com orientação ao paciente quanto à dieta não cariogênica e à correta higienização oral ${ }^{4-6}$. Esses pacientes devem ter acompanhamento regular, com profilaxia e cuidados bucais para controlar a doença periodontal e prevenir a perda dentária ${ }^{5-6,9}$.

\section{Conclusão}

A DCOF é uma patologia não incomum dos maxilares, desconhecida por muitos cirurgiões dentistas. Os casos clínicos apresentados ressaltam a importância dos exames por imagem como a TCFC no diagnóstico das lesões fibro-ósseas, que, como ilustrado, podem apresentar características imaginológicas bastante distintas. Informações clínicas como idade, etnia e condição dos dentes envolvidos são importantes no estabelecimento do diagnóstico, assim como acompanhamento em longo prazo.

\section{Abstract}

Objective: this study aims to report two clinical cases of florid cemento-osseous dysplasia (FCOD) with distinct characteristics in cone beam computed tomography $(C B C T)$ imaging. Case report: the first case was a 49 yearold woman with melanoderma, presenting asymptomatic hypodense lesions distributed throughout the mandible, suggestive of early-stage FCOD. The second case was a 48-year-old woman with melanoderma, presenting asymptomatic bilateral hyperdense lesions with hypodense halo in the mandible, characterizing the mature stage of FCOD. In both cases, the diagnosis of FCOD was established through the CBCT images associated with the clini- cal characteristics of the patients. No treatment was instituted, only periodic follow-up visits. Final considerations: the clinical cases reported highlight the importance of CBCT for diagnosing fibro-osseous lesions, which as illustrated, may present rather distinct imaging characteristics. Florid cemento-osseous dysplasia has a wide spectrum of presentation forms and it is found in imaging examinations conducted for other purposes; hence, it may lead the dental surgeon to make hurried decisions and often, inappropriate attitude choices, provided that surgical procedures are contraindicated in these cases.

Keywords: Maxillomandibular diseases. Cone beam computed tomography. Oral cavity.

\section{Referências}

1. Bencharit S, Schardt-Sacco D, Zuniga JR, Minsley GE. Surgical and prosthodontic rehabilitation for a patient with aggressive florid cemento-osseous dysplasia: a clinical report. J Prosthet Dent 2003; 90(3):220-4.

2. Sarmento DJ, Monteiro BV, de Medeiros AM, da Silveira EJ. Severe florid cemento-osseous dysplasia: a case report treated conservatively and literature review. Oral Maxillofac Surg 2013; 17(1):43-6.

3. Jardim ECG, Santiago Júnior JF, Guastaldi FPS, Magro Filho O, Garcia Júnior IR, Jardim Júnior EG . Displasia cemeto óssea florida - relato de caso. Rev Odontol Araçatuba 2010;31(2):31-4

4. Minhas G, Hodge T, Gill DS. Orthodontic treatment and cemento-osseous dysplasia: a case report. J Orthod 2008; 35(2):90-5.

5. Póvoa RCS, Nova AB, Maciel RM, Ramos MEB, Israel MS. Florid cemento-osseous dysplasia: case report. Ciencia Atual 2014; 4(2):2-7.

6. Consolaro A. Florid cemento-osseous dysplasia: one of the few contraindications to osseointegrated implants. Dental Press Implantol 2015; 9(1):26-33.

7. Gonçaves M, Pispico R, Alves FA, Lugão CEB, Gonçalves A. Clinical radiographic, biochemical and histological finding of florid cemento osseous dysplasia and reporto $f$ a casa. Braz Dent J. 2005;16(1):247-50

8. Das BK, Das SN, Gupta A, Nayak S. Florid cemento-osseous dysplasia. J Oral Maxillofac Pathol 2013; 17(1):150.

9. Kutluay Köklü H, Çankal DA, Bozkaya S, Ergün G, Bar E. Florid cemento-osseous dysplasia: report of a case documented with clinical, radiographic, biochemical and histological findings. J Clin Exp Dent 2013; 5(1):58-61.

10. Yildirim E, Bağlar S, Ciftci ME, Ozcan E. Florid cemento-osseous dysplasia: A rare case report evaluated with cone-beam computed tomography. J Oral Maxillofac Pathol $2016 ; 20(2): 329$.

\section{Endereço para correspondência:}

Tamara Fernandes de Castro

Avenida Doutor Mário Clapier Urbinatti, no 332 ,

Ap. 201, bairro: Zona 7

CEP 87020-260 Maringá, Paraná, Brasil

Fone: (44) 9986-79675

E-mail: tamara_tfc@hotmail.com

Recebido: 02/07/2017. Aceito: 31/07/2017. 